\title{
Measure the Evolution of Interbank Network Structure: Panel Data Analysis to Shibor Network
}

\author{
$\mathrm{Li} \mathrm{Li}$ and Chen Bing \\ School of Business Information \\ Shanghai University of International Business and Economics \\ Shanghai, China \\ School of Finance \\ Shanghai Lixin University of Commerce \\ Shanghai, China \\ lilycb163@163.com, chenbingfp168@163.com
}

\begin{abstract}
This paper constructs interbank network based on the overnight interest rates of SHIBOR database. Direct friction and indirect friction of interbank network are defined and the influencing factors of friction are tested through panel data analysis. Results of panel regression show that four network centrality measurements of in degree, out degree, in degree and out degree all have significant effect on indirect friction, by contrast, only in degree and out degree have significant effect on direct friction. There are different effects under the condition of different samples. The results indicate that network structure determined by price spread influences the relationship between banks and frictions.
\end{abstract}

Keywords: interbank network; network structure; direct friction; indirect friction; panel data analysis

\section{Introduction}

With the development of the financial liberalization reform and financial globalization, direct link between banks formed by lending to each other is becoming more closely, and it makes the modern banking system evolving into increasingly complex banking network [1-3]. The link of banking network has two different effects, one is to provide liquidity insurance mechanisms to achieve risk sharing, the other is to offer risk contagion channel-once a bank in the network collapse, shock will transmit to the entire network [4]. As a typical example, the link of the modern banking system is widely viewed as a key contributing factor to the 2008 global financial crisis [5].

In order to better understand the risk contagion mechanism of banking network, in the past ten years, many studies construct banking network based on interbank bilateral lending data of a national banking system, and simulate the contagion effects of bank failure shocks [6-13]. Researchers of IMF and BIS have paid more attention to crossborder risk contagion after the subprime crisis. Based on balance sheet network analysis and the inter-state banking system lending data of BIS, risk contagion effect of crossborder banking network under different shocks is simulated $[14,15]$.

In addition, complex network analysis has become a useful tool to investigate financial system. Some literatures examine the contagion effects under different network structure from the perspective of complex network. For example, reference [16] and reference [17] found that scale-free network has characteristics of robust and fragile. Reference [18] studied contagious effects based on the Erdös-Renyi random network. Particularly, the topological properties of a wide variety of financial systems around the world, both at the 
national level and at the level of cross-border transactions, have already been identified [19].

Based on reference [20], by linear probability models, reference [21] indicates social connections facilitate business connections and shows that connected banks partner more often appear in the syndicated loan market and that central banks in the network play dominant roles in various interbank transactions. When it comes to interbank exchange network, it has two functions that one is to make lending possibility and another is prevent interbank exchange. Friction in the financial markets refers to the difficulty of trading financial assets. It can be measured by the best holding time trading a certain amount of financial assets [22] or required price concessions of real-time exchange [23, 24]. Bid-ask spread are a determination method of market friction. Reference [25] defines direct friction and indirect friction to describe exchange difficulty of exchange network which possesses two different nodes--buyers and sellers and the network is undirected. Definitions are extended to directed network since interbank network is directed for each bank may lend and borrow money at the same time.

Different from the above research, this paper focuses on the influence of different network indicators to trade friction and liquidity based on network of interbank lending trading. Research questions of the paper are: how to measure financial network difficulties to liquidity and how these measurements affected by network structure features? There are two main contributions of this paper. Firstly, instead of frictions of undirected network, direct friction and indirect friction in directed network are defined to describe liquidity difficulties of interbank network which is directed network. Secondly, the factors which affect friction are tested by panel regression analysis based on the trading data between banks other than reference [20,21].

This paper is organized as follows. Section 2 is about interbank network construction and shows some basic feature along time of those networks. In Section 3, frictions of interbank network are defined and their variation along time is showed. Process and results of panel data analysis are put forward in Section 4. Conclusions are drawn in Section 5 .

\section{Interbank Network Construction}

\subsection{Data Description}

China interbank market is developing rapidly in the past 20 years. It has become China's biggest financial market in terms of trading volume. Given the huge numbers of interbank market trading members, at the same time, the trading volume of a few big banks have decisive influence on the interbank interest rates. There are 18 representative banks in SHIBOR which offered their daily bid and ask prices (same bank provides two same prices) since Dec 4, 2012. The sample banks including: Industrial and Commercial Bank of China-ICBC, The Agricultural Bank of China-ABC, Bank of China-BC, China Construction Bank-CCB, Bank of Communications-BC, China Merchants Bank-CMB, China Citic Bank-CB, China Everbright Bank-CEB, Industrial Bank-IB, Shanghai Pudong Development Bank-SPDB, Bank of Beijing-BB, Bank of Shanghai-BS, HSBC, Standard Chartered Bank of China-SCBC, Huaxia Bank-HB, Guangdong Development Bank-GDB, The Postal Savings Bank of China-PSBC and China Development BankCDB.

China interbank lending can be divided into 11 varieties according to time different, there are overnight, 1 week, 2 weeks, 3 weeks, 1 month, 2 months, 3 months, 4 months, 6 months, 9 months and 1 year. Among them, overnight trading volume is much larger than all other varieties. For example, trading volume of overnight accounted for the proportion of all transactions amount to $85.76 \%$ in June 2014. With data in SHIBOR database from Dec 4, 2012 to May 30, 2014, 373 days overnight (O/N) average prices are showed in Figure 1. 


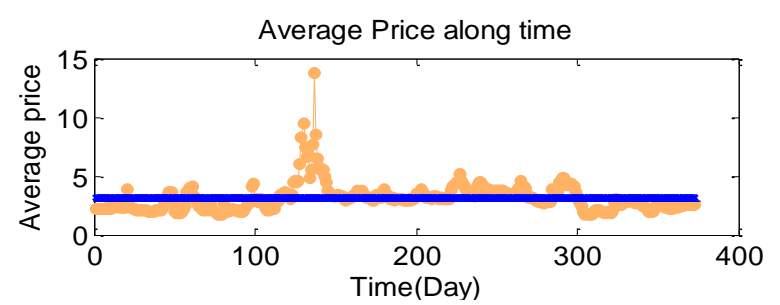

Figure 1. Total and Daily Average Price along Time

Daily average price fluctuates around total average price which is $3.0977 \%$. The maximum price is 18 on June 20, 2013, bid and ask by China Construction Bank. Average price of that day is $13.708 \%$, is the maximum daily average price. Chinese banks faced money shortage at those days and strong money demand lead to high price. The correlation coefficients between 18 banks are all greater than 0.98 indicates that almost all banks encountered same situation and employ similar price strategies. That means new perspective is needed to gain more information and new evidences from these data.

\subsection{Interbank Network}

According to 373 days overnight $(\mathrm{O} / \mathrm{N})$ spread, 373 direct networks of 18 nodes are built and each node denotes one of 18 banks. At day $t$, add a link from node $i$ to node $j(i \neq j)$ on network $k(t)$ where bid price of bank $i$ is greater than ask price of bank $j$, that means bank $i$ may borrow money from bank $j$. The direction of arrow is from $i$ to $j$ and in degree of $i$ increased by 1 as well as out degree of $j$ increased by 1 . There are several nodes have no links with the whole network which is isolated nodes. Some of their out degree or in degree equal to zero in directed network. Out degree of node $i$ is zero means bank $i$ can't borrow money from any other banks since its bid price is lower than ask price of other banks. In degree is zero means that bank may not lend money to others for its higher ask price. Density of each network is total degree divided by $n(n-1)$ ( $n=18$, the number of nodes). Figure 2(a) and Figure 2(b) show the ratio of nodes which have at least one link to other nodes along time.

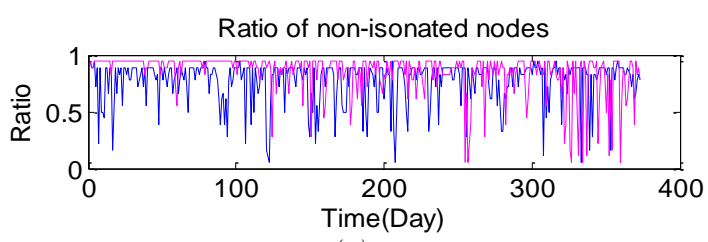

(a)

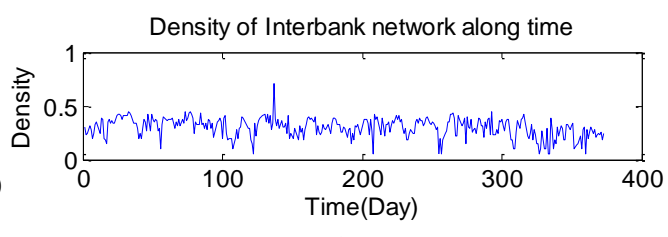

(b)

Figure 2. Interbank Network Density along Time

Figure 2(a) (blue: in degree greater than 0; pink: out degree greater than 0) indicates that the number of isolated nodes are decreased more frequently. Figure 2(b) is the network density along time is not over 0.5 except the most money shortage day, is 0.7059 . The distribution between $(1,0.5,0.4,0.3,0.2,0.1,0)$ is $(1,36,171,106,59,10)$. The correlation coefficients between these 3 measurements and average are not significant.

To one node in each network, the number of out degree means how many other banks one bank may borrow money from according to the spreads. On the one hand, the greater the out degree, the worse current liquidity it owns. The greater the out degree, the better potential liquidity it owns with higher financing costs on the other hand. The number of in degree means how many other banks one bank may lend money to according to the spreads. The greater the in degree implies more other banks may borrow money from that 
bank. Figure3 shows network structures on different date with nodes scale corresponding to in degree (left) and out degree (right).

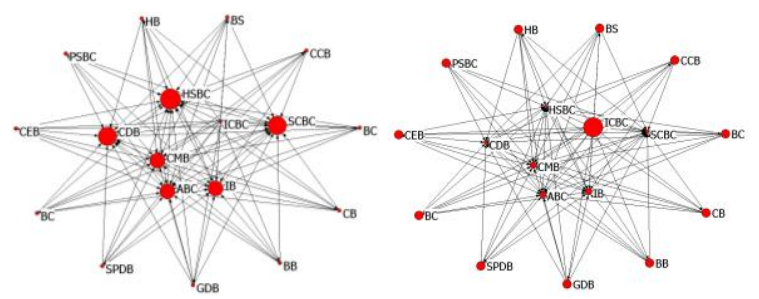

(a) Networks on January 4, 2012

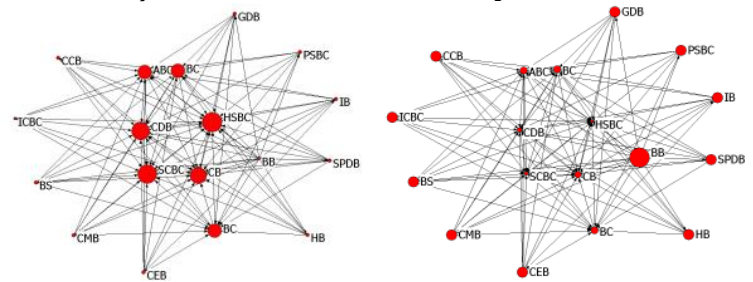

(b) Networks on January 4, 2013

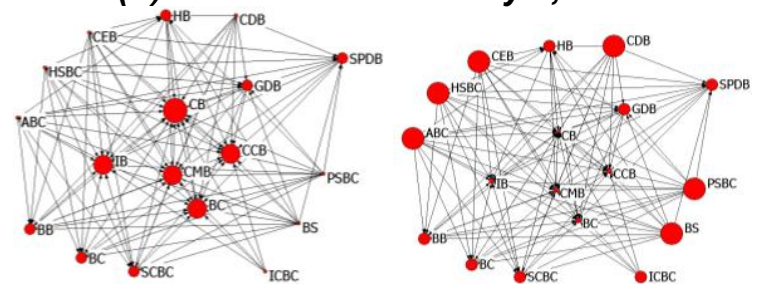

(c) Networks on January 2, 2014

Figure 3. Direct Networks at the Beginning of the Year (Left: Nodes Weighted by in Degree; Right: Nodes Weighted by Out Degree)

Figure 3 indicates that banks became more interconnected at the beginning of the year. Nodes scaled by in degree indicate more core-peripheral structure with larger nodes in the center of network than that by out degree. ICBC (Industrial and Commercial Bank of China) needs more money at the beginning of 2012 and BB (Bank of Beijing) on the same situation at the beginning of 2013. There are 6 banks lack money at the beginning of 2014. They are ABC (The Agricultural Bank of China), HSBC, CEB (China Everbright Bank), CDB(China Development Bank), PSBC(The Postal Savings Bank of China) and BS(Bank of Shanghai) .

Figure4 shows the network structures of the most money shortage day, June 20, 2013.

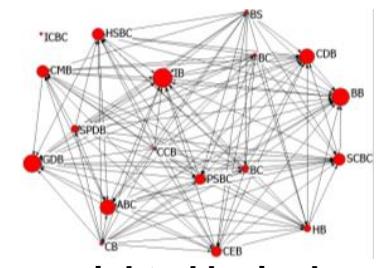

(a) Nodes weighted by in degree

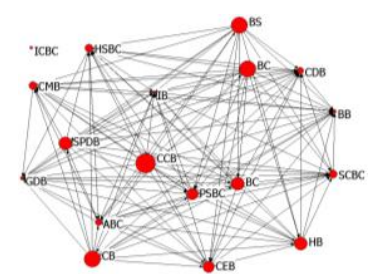

(b) Nodes weighted by out degree

Figure 4. Direct Networks on June 20, 2013

It is obvious that there are more links in the networks and network density is lager than ever. Form Figure 4(a), IB(Industrial Bank) can lend money to more other banks connected to it. Figure 4(b) indicates CCB(China Construction Bank) can borrow money from more banks, then $\mathrm{CB}, \mathrm{BC}$ and $\mathrm{BS}$ in the 2 nd position.

\section{Frictions of Interbank Network}




\subsection{Definitions to Interbank Network Frictions}

Direct friction $F_{i}(t)$ of node $i$ at time $t$ defined as the ratio between node $i$ 's out degree and it's degree.

$$
F_{i}(t)=\left\{\begin{array}{cl}
1 & \left|D_{i}(t)\right|=0 \\
\frac{\left|S_{i}(t)\right|}{\left|D_{i}(t)\right|} & \left|D_{i}(t)\right|>0
\end{array}\right.
$$

Where $\left|D_{i}(t)\right|$ denoted the number of node $i$ 's neighbor-set and $\left|N_{i}(t)\right|$ denoted the number of nodes in $i$ 's neighbor-set possesses links pointing to node $i$. $\left|S_{i}(t)\right|=\left|D_{i}(t)\right|-\left|N_{i}(t)\right|$ is the number of links that from node $i$ to it's numbers. $F_{i}(t)$ of a node equals to 0 while it is isolated or it's out degree is $0 . F_{i}(t)$ means the possibility node $i$ can lending from it's neighbors.

Indirect friction of node ${ }^{i}$ at time ${ }^{t}$ defined as

$$
I_{i}(t)=\left\{\begin{array}{cl}
1 & F_{i}(t)=1 \\
0 & \exists j \in N_{i}(t) \text { and }\left|D_{j}(t)\right|=1 \\
\frac{\sum_{j \in D N_{i}(t)}\left(\left|N_{j}(t)\right|-1\right) /\left|D_{j}(t)\right|}{\left|N_{i}(t)\right|} & \text { otherwise }
\end{array}\right.
$$

Average direct friction of $K$ at time $\mathrm{t}$ defined as

$$
A_{F}(t)=\sum_{i=1}^{|K(t)|} F_{i}(t) /|K(t)|
$$

Where $|K(t)|$ is the number of nodes of network $K(t)$.

Average indirect friction of $K$ at time ${ }^{t}$ defined as

$$
A_{I}(t)=\sum_{i=1}^{|K(t)|} I_{i}(t) /|K(t)|
$$

\subsection{Network Structure Affects Liquidity}

Liquidity to individual bank and whole interbank markets is important to the development of financial system as so to the whole economic system. Direct friction indicates how difficult a bank has to meet counterparty. The more out degree it possesses, the stronger bargaining power it will have and the more likely it can borrow money with lower price. It will face competitors if a bank possesses high indirect friction. That means his counterparties have more in degrees with more strong bargaining power and higher prices.

Direct friction describes difficulty to meet counterpart and indirect friction describes competitive situation. To a certain network, average direct friction indicates inactive interbank market and indirect friction indicates more active interbank market with more uncertainty.

\subsection{Interbank Network Frictions along Time}

Compute direct and indirect friction of each interbank network with (1) and (2). Average friction along time is shown at Figure 5. 


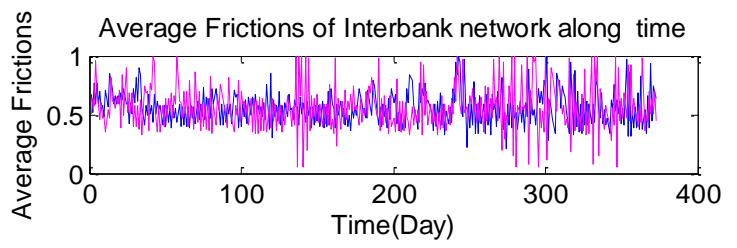

Figure 5. Average Frictions of Interbank Networks along Time

In Figure 5 (blue: average direct friction; pink: average indirect friction), mean of both average frictions are around 0.5 and sometimes reached 1. Fluctuation of average indirect friction is greater than that of average direct friction. Indirect frictions fluctuate wildly with high frequency around the most money shortage day. Few isolated nodes, high density and higher prices mean too active market. Average direct friction and average indirect friction increased at the same time between the 200th day and 300th day with lower out degrees, lower densities and prices about 3 . Lower in and out degrees, lower density and below average price lead to fluctuations during later period.

Computing the correlation coefficients between prices, in degree and out degree of every node, only that between direct friction and direct friction as well as that between indirect friction and out degree is statistically significant, respectively 0.61103 and 0.76358 . All 4 measurements have no correlation with prices.

\section{Results of Panel Data Analysis}

\subsection{Variable and Period Selection}

Reference [20-21] modeled banking network by undirected graph, four measurements of betweenness, eigenvector, closeness and degree are employed in the models. By contrast, In degree and out degree are considered in our research since Shibor network are directed. Betweenness, eigenvector, closeness centrality are computed too, but most of them are zeros since lower network density (see Figure 2).

Dependent variables are direct-friction and indirect-friction respectively and independent variable includes four indicators of in degree, out degree, centrality of in degree and centrality of out degree which divided by $n(n-1)$.

Keeping 18 samples bank unchanged, there are a total of 6714 trading data of 373 trading days from December 42012 to May 30 2014. Among them, there are 15 trading days price data which is too high because of money shortage of the middle of the years from June 6 to June 27 2013, there are a total of 6444 trading data after eliminate the 15 trading days data. Next, 6714 and 6444 trading data are regressed respectively following.

Network structure measurements are computed with networkx package, pythou2.7 for windows 7. Eviews 6.0 is employed for serials test and panel data regression.

\subsection{Unit Root Test}

To avoid spurious regression problem derived from data non-stationary, data stationarity must be tested before regression. The most commonly used test method is unit root test (Fisher-ADF test).

The null hypothesis of Fisher-ADF test is existing unit root (data non-stationary). Results of two samples are presented in Table I. 
Table I. Fisher-ADF test (I)

\begin{tabular}{|l|l|l|l|l|}
\hline \multicolumn{1}{|c|}{ Series } & \multicolumn{1}{c|}{ Sample } & \multicolumn{1}{c|}{ Statistic(P value) } & Sample & Statistic(P value) \\
\hline Direct_friction & 373 & $92.3803(0.0000)$ & 358 & $89.4451(0.0000)$ \\
\hline Indirect_friction & 373 & $173.917(0.0000)$ & 358 & $169.428(0.0000)$ \\
\hline In_degree & 373 & $226.231(0.0000)$ & 358 & $204.386(0.0000)$ \\
\hline Out_degree & 373 & $206.298(0.0000)$ & 358 & $217.539(0.0000)$ \\
\hline Ind & 373 & $286.227(0.0000)$ & 358 & $275.698(0.0000)$ \\
\hline Outd & 373 & $117.581(0.0000)$ & 358 & $114.414(0.0000)$ \\
\hline
\end{tabular}

According to Table I, Direct_friction, Indirect_friction, In_degree, Out_degree, Ind and Outd all refuse null hypothesis, that means the six series are all stationary data and the regression is not spurious.

\subsection{Cointegration Test}

Cointegration means that different variables has common stochastic trend. Cointegration test shows that there is long-term stable equilibrium relationship between variables, the regression residual of equation is stationary, the regression results are more accurate.

Unit root test results show that six variables are zero order single integer $I(0)$. So the cointegration test can be done to examine whether there is a long-term equilibrium relationship between variables. Cointegration test are conducted between dependent variables (Direct_friction and Indirect_friction) and independent variables (In_degree, Out_degree, Ind, Outd) under the two kinds of samples (373 and 358) respectively. With Kao test to each independent variable and two dependent variables, both samples have seven pairs of relationship are significant (see Table II). There are insufficient number of observations and Johansen test is adopted to deal with them (see Table III).

Table II. Kao Test Results

\begin{tabular}{|l|l|l|l|c|}
\hline \multicolumn{1}{|c|}{ Series } & Sample & t-Statistic(P value) & Sample & t-Statistic(P value) \\
\hline Direct_friction In_degree & 373 & $-4.593355(0.0000)$ & 358 & non-significant \\
\hline Direct_friction Ind & 373 & $-13.15797(0.0000)$ & 358 & $-12.48749(0.0000)$ \\
\hline Direct_friction Out_degree & 373 & non-significant & 358 & $-4.133381(0.0000)$ \\
\hline Direct_friction Outd & 373 & $-12.55062(0.0000)$ & 358 & $-14.51241(0.0000)$ \\
\hline Indirect_friction In_degree & 373 & $-5.994093(0.0000)$ & 358 & $-8.887701(0.0000)$ \\
\hline Indirect_friction Ind & 373 & $-19.85481(0.0000)$ & 358 & $-17.77332(0.0000)$ \\
\hline Indirect_friction Out_degree & 373 & $-14.70951(0.0000)$ & 358 & $-5.585104(0.0000)$ \\
\hline Indirect_friction Outd & 373 & $-4.372769(0.0000)$ & 358 & $-4.463556(0.0000)$ \\
\hline
\end{tabular}

Table III. Johansen Test of Results

\begin{tabular}{|l|l|l|l|}
\hline Series & $\begin{array}{l}\text { NULL } \\
\text { Hypothesis }\end{array}$ & $\begin{array}{l}\text { Trace } \\
\text { (P value) }\end{array}$ & $\begin{array}{l}\text { Max-eigen } \\
\text { (P value) }\end{array}$ \\
\hline $\begin{array}{l}\text { Direct_friction } \\
\text { Out_degree (373) }\end{array}$ & None & $315.6(0.0000)$ & $320.3(0.0000)$ \\
\cline { 2 - 4 } & At most 1 & $360.0(0.0000)$ & $360.0(0.0000)$ \\
\hline $\begin{array}{l}\text { Direct_friction } \\
\text { In_degree(358) }\end{array}$ & None & $326.5(0.0000)$ & $323.7(0.0000)$ \\
\cline { 2 - 4 } & At most 1 & $127.3(0.0000)$ & $127.3(0.0000)$ \\
\hline
\end{tabular}

According to Table II and III, there exists long-term equilibrium relationship between dependent variables (Direct_friction and Indirect_friction) and independent variables (In_degree, Out_degree, Ind, Outd).

\subsection{Model Selection and Regression Results}

Usually, there are three different models for panel data.

(1) Pooled Regression Model.

$$
y_{i t}=\alpha+X_{i t}^{\prime} \beta+\varepsilon_{i t}
$$

In this instance, ordinary least squares provides consistent and efficient estimates of the common $\alpha$ and the slope vector $\beta$. 
(2) Fixed Effects Model.

$$
y_{i t}=\alpha_{i}+X_{i t}^{\prime} \beta+\varepsilon_{i t}
$$

Where $\alpha_{i}$ embodies all the observable effects and specifies an estimable conditional mean. This fixed effects approach takes $\alpha_{i}$ to be a group-specific constant term in the regression model. It should be noted that the term "fixed" as used here signifies the correlation of $c_{i}$ and $X_{i t}$, not that $c_{i}$ is non-stochastic.

(3) Random Effects Model.

If the unobserved individual heterogeneity, however formulated, can be assumed to be uncorrelated with the included variables, then the model may be formulated as

$$
y_{i t}=X_{i t}^{\prime} \beta+\alpha+u_{i}+\varepsilon_{i t}
$$

That is, as a linear regression model with a compound disturbance that may be consistently, albeit inefficiently, estimated by least squares. This random effects approach specifies that $u_{i}$ is a group-specific random element, similar to $\varepsilon_{i t}$ except that for each group, there is but a single draw that enters the regression identically in each period.

In terms of the model choice, $F$ test is often used to decide to choose pooled model or fixed effects model, Hausman test is used to determine to choose random effects model or fixed effects model. Because there is no significant difference between different individuals from the time dimension and different cross-section from the cross-section dimension, and combining with the Hausman test and F test results at the same time, pooled regression model is chosen [26].

Parameters are estimated with least squares (LS) after the panel data directly mixed together. Estimated results from Table IV and Table V show that under two kinds of samples, Ind, Outd has a significant effect on Direct_friction. In_degree, Out_degree, Ind, Outd each has a significant effect on Indirect_friction.

And under the different samples, In_degree and Out_degree have opposite direction of impact on Indirect_friction and Direct_friction.

Table IV. Pooled Regression Results with Dependent Variable

\begin{tabular}{|c|c|c|c|c|c|c|c|}
\hline & \multicolumn{3}{|c|}{373 DAYS } & \multicolumn{4}{|c|}{358 DAYS } \\
\hline Variable & Coefficient & t-Statistic & Prob. & Coeffi & cient & t-Statistic & Prob. \\
\hline C & 0.736461 & 176.8816 & 0.0000 & & 0.539337 & 171.9355 & 0.0000 \\
\hline Ind & 0.011940 & 57.76089 & 0.0000 & & 0.025987 & -120.9925 & 0.0000 \\
\hline Outd & -0.024351 & -127.9753 & 0.0000 & & -0.039388 & 58.26050 & 0.0000 \\
\hline In_degree & -0.001977 & -0.472167 & 0.6368 & & -0.007865 & -1.517255 & 0.1292 \\
\hline Out_degree & -0.003744 & -0.518686 & 0.6040 & & -0.041122 & -1.002255 & 0.3162 \\
\hline R-squared & 0.242655 & of regression & $0.292544 \mathbf{I}$ & uared & 0.238692 & E. of regression & 0.293373 \\
\hline D. W. stat & 1.587298 & hwarz criterion & $0.380037 \mathbf{I}$ & W. stat & 1.573515 & chwarz criterion & 0.385718 \\
\hline
\end{tabular}
Direct_friction

Table V. Pooled Regression Results with Dependent Variable

\begin{tabular}{|c|c|c|c|c|c|c|c|}
\hline & \multicolumn{3}{|c|}{373 DAYS } & \multicolumn{4}{|c|}{358 DAYS } \\
\hline Variable & Coefficient & t-Statistic & Prob. & Coeffi & icient & t-Statistic & Prob. \\
\hline C & 0.539337 & 193.1149 & 0.0000 & & 0.534458 & 188.5797 & 0.0000 \\
\hline Ind & 0.025987 & 187.4122 & 0.0000 & & -0.039350 & -301.1074 & 0.0000 \\
\hline Outd & -0.039388 & -308.5957 & 0.0000 & & 0.027445 & 190.8310 & 0.0000 \\
\hline In_degree & -0.007865 & -2.800642 & 0.0051 & & -0.008378 & -2.948001 & 0.0032 \\
\hline Out_degree & -0.041122 & -8.492831 & 0.0000 & & -0.043487 & -8.877714 & 0.0000 \\
\hline R-squared & $0.690430 \mathrm{~s}$ & of regression & 0.196231 I & R-squared & 0.698118 & E. of regression & 0.194664 \\
\hline D. W. stat & $1.644510 \mathrm{~S}$ & hwarz criterion & -0.418605 I & D. W. stat & 1.636957 & chwarz criterion & -0.434628 \\
\hline
\end{tabular}
Indirect_friction

Even In_degree, Out_degree are greater than Ind and Outd, the effect of later two independent variables to directed friction are still significant. That implies relative closeness is more important to influence exchange difficulty than absolute degree. 
Indirect friction of one node depends on its neighbors and its neighbors' neighbors. In_degree and Out_degree are absolute number of its degrees and Ind and Outd are standardized by $n(n-1)$. Both results of 373 days and 358 days which extreme values eliminated are significant indicates that local structure of one node has influence on its competence on inter-bank borrowing so as to its liquidity difficulty.

With original data of 373 days, Ind plays positive effect and Outd plays negative effect. But when extreme values are eliminated and the series are more stationary, Ind plays negative effect and Outd plays positive effect. The reversion depended on the extreme values where more network density leads to the increasing of indirect friction as well as directed friction [25].

\section{Conclusion}

Firstly, this paper constructs banking network based on the overnight interest rates of SHIBOR database. According to the bid and ask data of 18 banks, 373 interbank directed networks each including 18 nodes are constructed. Network density is varied with the prices along time. Money shortage aroused while almost all banks need money at the same time and employ similar price strategies. Network visualization can present structure intuitionally and structure measurements can help identify features of banking system. Central bank may use such information in its regulation. Some networks emerge core-peripheral structure while some banks are very lack of money. When such kind of structure emerges for a certain period, the possibility of liquidity risk contagion through the network to other banks out of the network will rise. Expansionary monetary policies such as reserve requirement ratio cutting and open market operation will be prepared to relieve liquidity stress. On the contrary, contractionary monetary policies will be implied where core-peripheral structure isn't obvious and network density is low.

Secondly, nodes frictions and network frictions are extended to directed network especially interbank network frictions. Direct friction describes the difficulty of nodes to meet counterpart and indirect friction to describe competitive situation. Compared to other fiction definitions in financial field, structural factors are involved in nodes' frictions definition. They characterize nodes' situation from 2 aspects according to its neighbor and neighbor's neighbor. Average frictions level is corresponded to the liquidity of bank market. Lower network density corresponds to higher average direct friction. Higher density corresponds to higher average indirect friction [25]. Network frictions are varied with the prices along time. Investigating individual and network level frictions can help enterprise; institute, market and central bank obtain more information so as to make better decision.

Finally, the influencing factors of friction are tested through panel regression. According to regression results, the four network indicators of in degree, out degree, in degree density and out degree density have significant effect on indirect friction and in degree along with out degree have significant effect on direct friction. And under the different samples, in degree and out degree have opposite direction of impact on indirect friction and direct friction. That is because network density increases during money shortage--network structure changed and network density increased substantially and rapidly has important influence on those frictions.

These interbank networks are generated from the spread of bid and ask prices. Network structure influences nodes' situation as well as the whole network liquidity. There are different effects under different samples. The results indicate that network structure determined by price spread influences the relationship between structure and frictions. Network is regarded as market in reference [27]. To find coordination mechanism from network instead of market itself or the relationship between government and market will bring new perspective and knowledge. When it comes to the interbank market, to find new evidences, more and deep investigation such as the relationship between other liquidity indicators and frictions, frictions and other network measurements as well as that 
between the detail action and frictions during money shortage periods, will be put forward to further the research.

\section{Acknowledgements}

This paper is supported by Humanities and Social Science Youth Fund Project of Chinese Ministry of Education (12YJC790004), Major Projects of Scientific Research Innovation of Shanghai Municipal Education Commission (12ZS184) and 085 Project of Shanghai.

\section{References}

[1] C. Minoiu and J. Reyes, IMF Working Paper, vol. 74, (2011).

[2] F. Allen and A. Babus, "Networks in finance", In: Kleindorfer, P., Wind, Y., Gunther, R. (Eds.), The Network Challenge: Strategy, Profit, and Risk in an Interlinked World, Wharton School Publishing, (2009).

[3] R. Moghadam and J.Viñals, IMF, (2010).

[4] F. Allen and D. Gale, Journal of Political Economy, vol. 1, no. 108, (2000).

[5] P. Glasserman and H. P. Young, Journal of Banking \& Finance, in press, (2014).

[6] C. Furfine, Journal of Money, Credit and Banking, vol. 1, no. 35, (2003).

[7] C. Upper and A. Worms, European Economic Review, vol. 4, no. 48, (2004).

[8] J. Müller, Journal of Financial Services Research, vol. 1, no. 29, (2006).

[9] P. Mistrulli, Bank of Italy Economic working papers, vol. 641, (2007).

[10] M. Boss, H. Elsinger, M. Summers and S. Thurner, Quantitative Finance, vol. 4, no. 6, (2004).

[11] S. Wells, Bank of England Working Paper, vol. 230, (2004).

[12] M.Toivanen, Bank of Finland Discussion Paper, vol. 6, (2009).

[13] H. Degryse and G. Nguyen, International Journal of Central Banking, vol. 2, no. 3, (2007).

[14] M. Espinosa-Vega and J. Solé, IMF Working Paper, vol. 105, (2010).

[15] J. Chan-Lau, IMF Working Paper, vol. 107, (2010).

[16] A. Haldane, Speech, Bank of England, (2010).

[17] P. Gai and S. Kapadia, Proceedings of the Royal Society A, vol. 2120, no. 466, (2010).

[18] E. Nier, J. Yang, T. Yorulmazer and A. Alentorn, Journal of Economic Dynamics and Control, vol. 1, no. 31, (2007).

[19] B. Spiros and A. Kirman, CESIFO Working Paper, vol. 4756, (2014).

[20] M. A. Ferreira and P. Matos, Review of Financial Studies, vol. 25, (2012).

[21] F. H. Joel, L. Jongsub and S. Felix, University of Florida, (2014).

[22] S. Lippman and J. McCall, American Economic Review, vol. 76, (1986).

[23] H. Stoll, Friction, The Journal of Finance, vol. 55, (2000).

[24] H. Demsetz, Quarterly Journal of Economics, vol. 82, (1968).

[25] L. Li, B. Wu, Z. Chen and L. Zhao, "Social network as double-edged Sword to Exchange Network", Frictions and The Emerging of Intellectual Intermediary Service. Complex(1)', (2009) February 23-25, Shanghai, China.

[26] M. Wooldridge, "Econometric Analysis of Cross Section and Panel Data", MIT Press, Cambridge, (2002).

[27] H. White, "Markets from Networks: Socio economic Models of Production", Princeton University Press, Princeton, (2002). 